\title{
Virtual Reflections and Virtual Shadows in Mixed Reality Environments
}

\author{
Frank Steinicke, Klaus Hinrichs, and Timo Ropinski \\ WWU Münster, Institut für Informatik, Einsteinstrasse 62, \\ 48419 Münster, Germany \\ \{fsteini, khh, ropinski\}@math.uni-muenster.de \\ http://viscg.uni-muenster.de
}

\begin{abstract}
In this paper we propose the concepts of virtual reflections, lights and shadows to enhance immersion in mixed reality (MR) environments, which focus on merging the real and the virtual world seamlessly. To improve immersion, we augment the virtual objects with real world information regarding the virtual reality (VR) system environment, e.g., CAVE, workbench etc. Realworld objects such as input devices or light sources as well as the position and posture of the user are used to simulate global illumination phenomena, e.g., users can see their own reflections and shadows on virtual objects. Besides the concepts and the implementation of this approach, we describe the system setup and an example application for this kind of advanced MR system environment.
\end{abstract}

\section{Introduction}

Seamlessly merging the real and the virtual world created within a computer is a challenging topic in current virtual reality (VR) research. Technology that superimposes the real world by computer-generated images is called augmented reality (AR) whereas the enhancement of virtual worlds using real-world data is called augmented virtuality (AV). In both cases, the goal is seamlessly merging of the real and virtual world. The term mixed reality (MR) ([3]) encompasses both augmented reality as well as augmented virtuality. As mentioned in [4], the main issues of MR environments are consistency of geometry, time, and illumination. Of course, one of the most important tasks is that superimposed objects have to be placed at the exact position where they would exist in the real world. Likewise, reflections, light sources and shadows must match in both worlds to obtain consistent global illumination; hence movements in the two worlds must be synchronized.

Recent approaches blend both synthetic as well as real objects to excellent quality images. However, the addressed global illumination is applicable only under specific limitations, e.g., non real-time performance, or if certain requirements are satisfied, e.g., a geometric computer model of the real scene is required or light sources need to be static [2]. The strategies proposed in [4] which improve the usage of virtual lights and shadows in MR use special hardware that is usually not accessible in most MR system environments. The effect of virtual reflections with respect to immersion in AR environments has been examined in [5]. This approach approximates reflections of real-world objects on virtual objects by extracting environment information from the background. 



Fig. 1. Captured video frame of the working area (left). Reflection from the user's point of view (right).

Our approach and system setup presented in this paper combines global illumination phenomena in MR system environments in order to blur the borders between the real and the virtual world. Hence we add visual information of the real environment surrounding the user to the virtual objects, e.g., objects dynamically mirror the environment, and thus users can see their own reflections and cast shadows in the virtual world. The proposed concepts and application scenario support the realization of an MR environment that provides a novel way of visual interaction.

In Section 2 we describe implementation and setup of our MR system environment. Section 3 presents an example application and discusses the benefits of our approach. Section 4 concludes the paper and gives an overview about future work.

\section{Mixed Reality Environment Setup}

\subsection{Virtual Reflections}

Reflective surfaces such as glass, metal or water reflect their environments. Usually an environment map is constructed in order to apply reflections in real-time computer graphics. For this purpose two types of maps have been proposed in [1]: spherical environment maps and cubic environment maps. In a spherical map a sphere with a single spherically distorted texture surrounds the virtual scene. Using cube maps the virtual environment is approximated by the six faces of a cube having an appropriate texture map; the cube is centered at the camera position. To simulate reflections rays are cast through the environment map. Green ([1]) preferred the usage of cubic environment maps because of the easier integration into 3D graphics hardware. Cubic environment maps are created by rendering a virtual scene or capturing real-world information with a 90-degree field of view (FoV) camera resulting in left, right, front, back, top and bottom textures. Since the textures are static images that are unaffected when the environment changes, interaction results in an inconvenient behavior in a highly interactive MR system environment. Thus, to further improve immersion in interactive MR applications changes of the real-world environment have to be considered. Hence a dynamic environment map representing the complete surrounding of the MR system environment is desirable. In our approach we simplify this idea and 
use a single USB camera that records the main working area of the user, i.e., the area in front of the screen in our projection-based MR system environment. The remaining areas, which are not subject to change, are given by static images of the surrounding generated by rendering a geometric model of the MR system environment.

In our setup the camera is attached to the top of a responsive workbench (RWB) (see Figure 2 (left)). Figure 1 (left) shows an image of a user in the working area captured from a live stream. This image is part of the cubic environment map containing the remaining areas. A resulting reflection based on this environment map is shown in Figure 1 (right). By using this approach, virtual objects with a reflective surface mirror other virtual objects and the static environment as well as the varying working area in front of the screen. Although the lighting conditions in MR system environments are often insufficient, users experience an adequate reflection (see Figure 1 (right)) because additional light is dispersed from the projection screen.

\subsection{Virtual Shadows}

Besides reflections the usage of virtual light sources and virtual shadows enhances the realism of virtual objects. In order to apply and modify direct lighting in MR environments in an intuitive way, we propose the following light interaction of real and virtual objects. Passive markers are attached to a real floor lamp for real-time tracking via our optical tracking system. Position and orientation of the tracked lamp are exploited to place the virtual light source accordingly. Thus a user simply moves the floor lamp, and the virtual light is positioned in the virtual scene in the same way as the real-world light source and illuminates the scene.

In the real world a user, moving in-between light source and an illuminated object, casts a shadow on the object. Because the user is usually not defined as geometry in the visualization system, it is difficult to calculate a corresponding shadow. In general, however, the user's head and at least one hand are tracked to allow an immersive direct interaction. In our approach we augment the virtual scene with a geometric model approximating the user's posture used for shadow generation. Therefore the depth information of this geometry is rendered into a depth texture, which is applied later on during shadow mapping. Using this approach no special hardware devices are necessary for shadow generation. The tracked floor lamp is illustrated in Figure 2 (left), while the resulting shadow of the user's hand in combination with the described reflection can be seen on the engine hood of the car in Figure 2 (right).

\section{Application Scenario}

To evaluate the described concepts we have implemented an example application that supports the advanced exploration of different car models (see Figure 2). The virtual cars can be illuminated intuitively by naturally positioning the previously described tracked floor lamp. The surrounding of the VR laboratory can be seen in the reflection and thus the surface structure of the car looks more realistic. The virtual car seen from the user's point of view is illustrated in Figure 2 (right), which shows clearly visible reflections and shadows on the engine hood. 

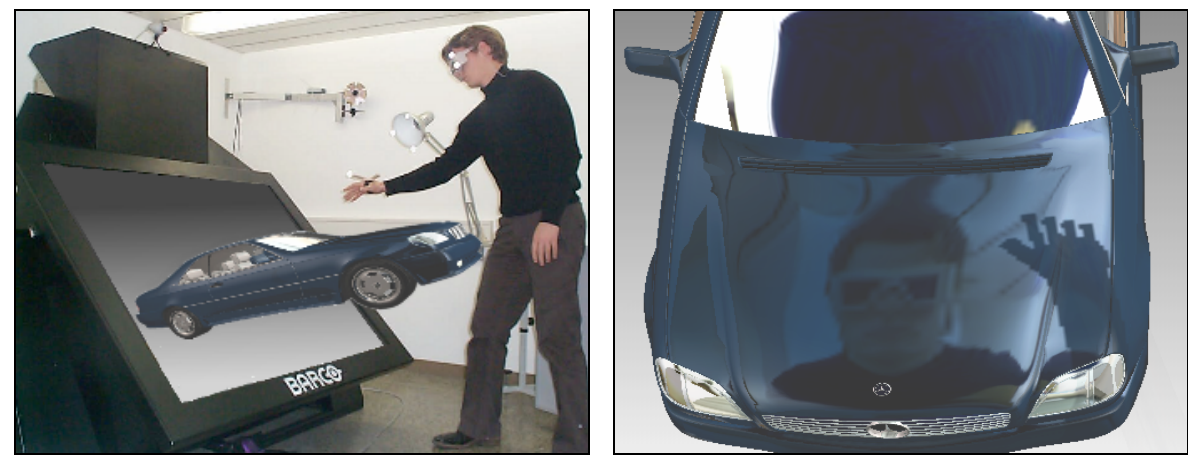

Fig. 2. Responsive workbench environment with tracked head, hand, and floor lamp (left). Reflections and shadows on the engine hood from the user's point of view (right).

\section{Conclusion and Future Work}

We have proposed the concepts of an advanced MR system environment that considerably increases realism of virtual objects as well as an example application which underlines the benefits. We are confident that the described approach enhances the VR-based design of cars in automotive industry since surface properties are displayed more realistic, and an intuitive positioning of light sources is possible.

An evaluation of the described system setup will show in how far the usage of virtual reflections and virtual shadows increases the users' immersion and improves object interaction. To further enhance this approach more high-resolution cameras could be attached to the setup, and additional information about the user's posture could be used to generate more precise shadow models.

\section{References}

1. Greene, N.: Environment Mapping and Other Applications of World Projections. IEEE Computer Graphics and Applications 6(11) (1986) pp. 21-29.

2. Jacobs, K., Loscos, C.: Classification of Illumination Methods for Mixed Reality. Eurographics 2004, State of the Art Report (2004) pp. 95-118.

3. Milgram, P., Kishino, F.: A Taxonomy of Mixed Reality Visual Displays. IEICE Trans. on Information Systems E77-D (12) (1994) pp. 1321-1329.

4. Naemura, T., Nitta, T., Mimura, A., Harashima, H.: Virtual Shadows in Mixed Reality Environment Using Flashlight-like Devices. Trans. of Virtual Reality Society of Japan 7(2) (2002) pp. 227-237.

5. Ropinski, T., Wachenfeld, S., Hinrichs, K.: Virtual Reflections for Augmented Reality Environments. Int. Conference on Artificial Reality and Telexistence (2004) pp. 311-318. 